\title{
La cysticercose bovine. Son importance dans les zones sahéliennes d'élevage de la République du Tchad.
}

\author{
por M. GRABER
}

La cysticercose ou ladrerie bovine est une affection caractérisée par la présence dans le muscle, le conjonctif intermusculaire ou les organes du bœuf et d'autres ruminants de cysticerques qui ne sont pas autre chose que les formes larvaires de Taenia saginata (Goeze 1782) de l'homme ou de Taenia hyaence (Baer 1924) de la hyène. Le cysticerque se nomme dans le premier cas Cysticercus bovis (Cobbold) ef dans le second cas Cysticercus dromedarii (Pellegrini 1945).

Cosmopolite, la ladrerie est une zoonose importante non seulement du fait des saisies considérables qu'elle entraine chaque année, mais encore par les conséquences fâcheuses qu'elle a sur l'un des hôtes définitifs, I'homme (téniasis et plus rarement cysticercose).

Il nous a paru intéressant dars cet article. to st en étudiant les données propres au Tchad, de jeter un coup d'œil sur ce qui a été fait et écrit en cette matière dans le monde au cours de ces vingts dernières années.

\section{HISTORIQUE}

Le petit nombre et la dispersion de Cysticercus bovis sur les carcasses, semble expliquer le fait que ce parasite soit passé longtemps inaperçus. Leuckart (57) pense néanmoins que le Taenia saginata existait déjà depuis la plus haute antiquité, mais qu'il était confondu avec 'e Taenia solium.

Les premiers travaux remontent à la fin du XVIII siècle. C'est Goeze (86) en 1782, qui établit une distinction assez précise entre Taenia solium et Taenia saginata : il qualifie le premier de translucide, aplati (pellucida) et le second de charnu, engraissé (saginata).

Leuckart, en novembre 1861, réussit à infesfer des veaux à partir de quatre-vingts anneaux de Taenia saginata. Cette transmission fut confirmée par de nombreux auteurs (Hertwig). L'expérience inverse : infestation de l'homme par Cysticercus bovis du boeuf, fut réalisée peu de temps après par Perroncito en Italie (1877) et Oliver aux Indes (1869), ces chercheurs servant eux-mêmes de matériel d'expérience.

Quant à Cysticercus dromedarii, il a été une première fois soupçonné par Martinaglia en 1932 aux abattoirs de Johannesburg : d'après Mönnig, les crochets ressemblaient fort à ceux de Taenia hyaenae (114). Aucun nom ne lui fut donné. II a été redécouvert et décrit chez les chameaux et les bovins de la Somalie italienne par Pellegrini en 1945 (74-75-76) ef son cycle évolutif mis en évidence par le même auteur en 1947 (77).

\section{MORPHOLOGIE, DEVELOPPEMENT ET CYCLE EVOLUTIF DES ESPECES EN CAUSE}

\section{a) Cysticercus bovis (Cobbold).}

Arrivé à son complet développement dans les muscies ou les organes des animaux atteints, ce cysticerque se présente sous la forme d'une vésicule oblongue de $7,5 \mathrm{~mm}$ à $9 \mathrm{~mm}$ sur $5,5 \mathrm{~mm}$ environ. Sa couleur est blanc gris ou brun rouge. Cette vésicule, entourée d'une membrane épaisse, est remplie d'un liquide plus ou moins clair au milieu duquel apparaît le plus

Reçu pour publication : mars 1959. 
souvent une tache blanc jaunâtre qui correspond à la tête invaginée ou scolex. Ce dernier, légèrement déprimé en son centre, est de très grande taille $(1,5 \mathrm{~mm}$ à $2 \mathrm{mr}$ de diamètre $)$ i il n'est pas armé : il n'existe ni crochets ni rostellum. Les quatre ventouses, autour desquelles se remarque quelquefois une zone de forte pigmentation noire, sont très fortement musclées permettant ainsi, à l'état adulte chez l'homme, une très forte adhérence à la paroi de l'inlestin, adhérence bien supérieure même à celle du Taenia solium bien que celui-ci dispose de crochets.

Le cysticerque vivant ingéré par un être humain (viandes crues ou mal cuites, souillures, etc.) s'évagine dans l'intestin au bout de vingtquatre heures, sous l'influence des sucs digestifs (bile surtout). Le scolex par ses ventouses s'attache à la muqueuse intestinale au voisinage du pylore. Le cestode grandit peu à peu à un rythme qui atteint environ $72 \mathrm{~mm}$ par jour (86), les plus jeunes anneaux repoussant les plus âgés vers l'arrière. Les premiers proglottis mûrs sont expulsés par le porteur de 60 à 70 jours après l'ingestion de viande ladre, plus tard ( 3 à 6 mois) selon certains auteurs (23).

Le parasite adulte, Taenia saginata, fait partie de la catégorie des grands cestodes : il mesure de 4 à 10 mètres, quelquefois plus 174 mètres - 86). Le scolex a les mêmes caractères que celui de Cysticercus bovis. Le cou est long et étroit. Les premiers anneaux sont courts, les autres augmentent progressivement de largeur et les anneaux gravides ont de 16 à $20 \mathrm{~mm}$ de long sur 4 à $7 \mathrm{~mm}$ de large. Ils sont rejetés seuls séparément, souvent dans l'intervalle des selles, ce qui es't extrêmement gênant. lls ren. ferment un utérus muni de 15 à 35 branches latérales de chaque côté. L'ensemble est bourré d'œufs ovoïles de 30 à $50 \mu$ sur 20 à $30 \mu_{1}$ chacun d'eux possédant un embryorl pourvu de 6 crochets.

Taenia saginata est sujet à de nombreuses anomalies qui ont trait à la coloration du parasite (Ténia ardoisé ou ténia algérien), au nombre de ventouses, à la présence de déux pores génitaux sur le même anneau ou à la perforation des anneaux (Ténia fenêtré).

La longévité du cestode est mal fixée : on connaît des individus qui l'ont hébergé pendant
20 ans, 35 ans (86) et 50 ans (11). Nous avons observé deux cas de téniasis humain à Taenia saginata rebelles à tout traitement : le parasite existait déjà depuis quatre ans et dix ans.

En ce qui concerne le nombre d'œufs émis, la question est très controversée. Selon Cobbold cité par Parlier (72), il faut tabler sur une évacuation de 400 proglottis mûrs par an. Chacun d'eux peut contenir 30.000 œufs, ce qui donnerait au total 140 millions d'œufs éliminés au cours d'une année par une seule personne. Pour Railliet (86), avec le nombre de proglottis, il n'y a que 8.000 œufs par anneau, soit pour une année 42 millions d'œufs seulement. Borchert (Talavera - 106), avance le chiffre de 80.000 œufs mûrs. Les quelques anneaux que nous avons pu examiner, ne paraissaient pas avoir plus de 10.000 œufs. Certains étaient encore moins riches. Une émission de 50 millions d'œufs par an nous semble constituer un maximum pour le Tchad.

Ces oufs absorbés par un ruminant, tombent dans l'intestin où la coque est dissoute. L'embryon, grâce à ses crochets, traverse la muqueuse intestinale et passe dans la circulation sanguine qui l'entraîne en divers points de l'organisme.

L'embryon perd ses crochets et croît selon un rythme qui a été étudié par Mclntosh (62) pour les quatre premières semaines de l'existence du parasite :

\section{TABLEAU I}

\begin{tabular}{|c|c|c|c|}
\hline $\begin{array}{c}\text { Nombre } \\
\text { de } \\
\text { jours }\end{array}$ & $\begin{array}{c}\text { Dimension totale } \\
\text { de la tache } \\
\text { ladrique (en mm) }\end{array}$ & $\begin{array}{c}\text { Dimension } \\
\text { dusticerque seur } \\
\text { (en rm) }\end{array}$ \\
\hline 11 & 2 à 3 & $0,13-0,15$ sur & $0,13-0,09$ \\
13 & 3 à 4 & $0,16-0,17$ sur & $0,10-0,13$ \\
20 & $6-10$ à $7-9$ & $1,30-1,65$ sur & $0,90-1,30$ \\
28 & $4-5$ à $6-8$ & $0,90-1,15$ sur & $0,80-1,18$ \\
\hline
\end{tabular}

A ce stade, le scolex commence à se former, mais il n'a pas encore de ventouses.

Hertwig (86-104) a précisé les stades suivants. Au bout de 18 semaines, la croissance du cysticerque est totalement achevée. On admet cependant que la cysticercose est identifiable à partir de la sixième semaine faisant suite à 
l'infestation. On relève alors les dimensions suivantes :

Ensemble de la lésion : 4,2 × 3,5 mm.

Cysticerque : $3 \times 2,5 \mathrm{~mm}$.

Scolex : $1 \times 1,3 \mathrm{~mm}$.

Cysticercus bovis subit dans des délais variables (un an pour la plupart des auteurs), une dégénérescence de type caséo-calcaire. II en résulte, dans certains cas, une atrophie progressive de la vésicule qui devient très petite, de la grosseur d'un grain de chanvre : c'est ce que l'on appelle la ladrerie sèche; elle est d'ailleurs rare au Tchad.

Cadéac (14), citant Ostertag, prútend même que les parasites sont capables de disparaitre avec le temps. Cette notion est très intéressante en ce sens qu'elle pourrait expliquer - comme nous le verrons plus loin - les différences considérables abservées au Tchad dans le taux d'infestation des jeunes et des adultes.

Enfin, it n'est pas exceptionnel de rencontrer sur le même animal des cysticerques vivants et des cysticerques morts ou dégénérés (102-114).

\section{b) Cysticercus dromedarii (Pellegrini 1945).}

II se présente (74 à 81 ) sous l'aspect d'un kyste ovoide (localisations musculaires) de 5 à $14 \mathrm{~mm}$ ou d'un kyste rond de 2 à $9 \mathrm{~mm}$ (foie. cerveau, organes lymphatiques). Certains cysticerques atteignent même des tailles imposantes (19 à $20 \mathrm{~mm})$. Le kyste, de couleur grise ou jaunâtre selon les espèces, contient un liquide limpide, incolore au milieu duquel se voit unc « masse » ronde, blanche qui occupe à pe: près toute la largeur du parasite.

Après ouverture et éclaircissement de la « marse » en question, on distingue à l'extrémité de celle-ci le scolex g'obuleux mesurant de 600 à $1.000 \mu$. Il porte quatre ventouses et un rostre cylindrique armé d'une double couronne de crochets (de 32 à 44). Les plus grands (de 187 à $218 \mu)$ possèdent un manche gros, droit et plus long que la lame; celle-ci est très large avec un arc dorsal peu courbé et un arc ventrai formant un angle obtus avec une garde longue. muriforme et fourchue, semble-t-il. Les petits crochets ont de 112 à $137 \mu$. Le manche plus court que la lame, est retourné dans le sens cpposé. La garde bien relevée est fourchue.
Nos observations à partir de quelques exempiaires de Cysticercus dromedarii coinncident à peu de chose près avec celles de Pellegrini tant du point de vue dimensions que du point de vue description des crochets.

Le reste de la « masse » est constitué par un « cou » et un corps relativement long (de 10 à $25 \mathrm{~mm}$ ) fortement imprégné de corpuscules calcaires.

Le cysticerque est entouré d'une membrane adventice qui adhère plus ou moins aux tissus voisirs. Cette adhérence qui est très forte dans certains cas (localisations musculaires). -'existe pas ailleurs, de sorte que le parasite s'énuclée facilement (foie-localisations méningées).

Cysíicercus dromedarii subit les mêmes phénomènes de dégénérescence que Cysticercus jovis.

Abzorbé par une hyène lanimaux morts jébris d'abattoirl. il se transforme dans l'intestin du carnivore et donne le Taenia hyaenae (Bee: 1924), dont nous donnons sommairement les caractères essentieis $\left(7^{\prime}-8-81\right)$ : c'est un cestode de taille moyenne $(3$ mètres à 3,50 mètres), robuste, blanc, peu transparent. La tête g'obuleuse, pigmentée, porte quatre ventouses en saillie $(400 \mu)$ et des crochets qui ont été décrits plus haut. Le cou est plus étroit que a tête. Les premiers anneaux se remarquent à 1-1.6 mm de l'extrémité du scolex; ils sont plus iarges que longs. Les proglottis mûrs sont plus longs que larges $(30 \times 6 \mathrm{~mm})$ avec des pores géritaux irrégulièrement alternes. L'utérus se ramifie en 24-30 branches latérales. Les œufs de forme ovoide $(25-29 \times 29-36 \mu)$, ont une coque эssez épaisse.

Ces œufs avalés par un hôte intermédiaire convenable (chameau - bovins - ruminants sauvages), passent dans l'intestin puis, par le système sanguin, gagnent les sièges de prédilection habitueis. Le cycle évolutif ladultes : hyène forme larvaire : ruminants) est a'ors « boucié ».

\section{ESPECES AFFECTEES}

\section{a) Cysticercus bovis.}

Nous l'avons rencontré au Tchad chez le bœuf, le zébu, le chameau, la gazelle (Gazella 
rufifrons et Gazellas dorcas) et diverses antilopes. Les ruminants sauvages ne sont donc pas épargnés ef l'infestation prend souvent chez eux une allure gravé : sur une gazelle abattue à Abougoudam (est tchadien), les cysticerques se touchaient presque dans les psoas. On ne saurait, en cette matière, trop insister sur le danger présenté par les viandes de chasse, abondamment consommées et rarement inspectées; le téniasis humain dans de nombreux cas n'a pas une autre origine.

Cysticercus bovis existe également chez l'homme à l'état larvaire : il provoque une cysticercose oculaire ou cérébrale. Cependant ce parasite est moins souvent en cause que Cysticercus cellulosae.

Nous avons eu la surprise par deux fois (1955 et 1956) de le mettre en évidence chez 'e mouton, l'absence de crochets, après éclaircissement, permettant un diagnostic facile.

On a signalé enfin sa présence chez la girafe (86) et chez le daim (114).

\section{b) Cysticercus dromedarii.}

Le parasite se retrouve chez le chameau, le zébu et la chèvre (81). Souvent Cysticercus bovis et Cysticercus dromedarii coexistent (21). Parmi les animaux sauvages, citons Cephalophus grimmi en Somalie (15).

Au Tchad, nous l'avons recueilli sur un dama. lisque (Damaliscus korrigum), une gazelle, un chameau et des zébus.

\section{REPARTITION GEOGRAPHIQUE}

\section{$1^{\circ}$ Cysticercus bovis.}

Il est cosmopolite : on l'a décelé à peu près dans tous les pays du monde, même en Australie et en Nouvelle-Zélande où sa présence est pourtant exceptionnelle.

a) En Europe, il faut distinguer :

$1{ }^{\circ}$ Les pays où la cysticercose bovine accuse une certaine progression depuis la guerre. Ce sont : l'Angleterre, l'Allemagne, la Hongrie, I'Italie, les Pays-Bas, la Suisse, le Danemark ef I'Islande (64).'
En Angleterre, 'l'incidence moyenne de la cysticercose, selon le ministère de l'Agriculture, est passée de 0,21 à 0,58 p. 100 de 1949 à 1953 (6-96-97). Dans 41 abattoirs, au cours des années 1952-53, le taux d'infestation oscillait autour de 1,03-3,47 p. 100. Ailleurs, les sondages effectués (39-43-61-85) ont montré des pourcentages de 0,3 à 6 (Blackpool - 1948-50). Dans 99 p. 100 des cas, il s'agissait d'infestations légères.

En Allemagne, la cysticercose bovine était déjà très répandue avant la dernière guerre. La proportion des bovins porteurs progresse de 0,36 p. 100 en 1951 à 0,52 p. 100 en 1955 et en Allemagne orientale de 0,49 p. 100 à 2,2 p. 100 de 1946 à 1952 (53). On signale même 1,62 p. 100 à Nuremberg en 1954 (103).

En Hongrie, en Italie, au Danemark, en Islande, en Hollande ef en Belgique, les pourcentages atteignent en moyenne, pour l'année 1956, $0,5,1,5,0,66,2,73,0,79,1,57$ et $1(38-64)$, avec çà et là des zones de plus fortes contam:nations (de 3,4 à 8 p. 100 à Pise : Pellegrino. 32).

$2^{\circ}$ Les pays où la cysticercose bovine est faible ou semble marquer une nette régression. Ce sont : certains régions de la Russie d'Europe, la France, l'Autriche, la Norvège, I'Espagne et le Portugal (3).

L'Ukraine et la Haute-Volga qui présentaient des taux voisins de 13 à 15 p. 100 (1943), paraissent se débarrasser de Cysticercus bovis à un rythme accáléré. Boiko (24) donne des chiffres probants pour les régions de laroslav et de Gorki où il n'y a plus actuellement que 0.1 p. 100 d'arimaux ladres. Par contre, la cysticercose demeure fréquente dans le nord et le nord-est du pays.

En France, où l'affection avait subi une forte poussée du fait de la guerre 0,5 p. 100 d'après Reuter - 90), la cysticercose est aujourd'hui relativement rare, de l'ordre de 0,01 p. 100 en 1956 aux abattoirs de la Seine (64). Cependant des chiffres plus importants ont été notés à Rouen $(0,68$ p. 100 sur des veaux - 8) et en Vendée (29). Il est probable qu'en France. comme dans bien des pays, il existe des zone: plus fortement touchées les unes que les autres. 
sous l'influence de facteurs divers (mauvaise hygiène notamment).

En Autriche, le pourcentage tambe de 0,1 à 0,03 (1955 à 1957) et en Norvège (1955-56). de 0,02 à $0,015(64)$.

$3^{\circ}$ Les pays sur lesquels nous ne possédons pas de renseignements très précis : la Roumanie $\{0,35$ p. 100 en 1956), la Tchécoslovaquie $(0,2$ à 0,5 p. 100$)$, la Pologne (de 0,01 à 0,6 p. 100 - 111). la Bulgarie (5.8 p. 100 - 13). En Yougoslavie, les pourcentages publiés varient de 0.01 à 30 (Montenegro : 38-56-84-91').

b) En Asie, on relève notamment le taux de 2 à 3 p. 100 pour la Kirgizie (66) et celui de 6.8 p. 100 pour la Mongolie extérieure (20). L'infection est faible au Japon $(0,27$ p. 100), au Cambodge, au Vietnam (46); elle existe en Malaisie (27). Elle est commune au Pakistan. En Iran, on trouve de 7 (26) à 50 p. 100 des animaux atteints selon les régions (1 10), au Liban 2 p. 100 et en Syrie 18 p. 100 (88). La Turquie (54) ef Israël. constituent également des pays de forte endémicité.

c) En Amérique : Grégoire (38) a rassemblé quelques renseignements concernant l'Amérique du Nord. Le taux est de 0.37 p. 100 aux U.S.A. et de 8 à 12 p. 100 au Mexique avec des pourcentages plus élevés dans les états où l'inspection des viandes n'est pas systématique (34).

En Amérique du Sud, on signale pour le Vénézuela 0,5 p. 100 à Caracas (63) et pour le Brésil de 1 p. 100 à 1,9 p. 100 à Sao-Paulo (71-91). La cysticercose n'est pas très rare en Argentine.

d) En Afrique, au cours de ces vingt dernières années, un important travail de recherche et de statistique a été mené à bien et l'on s'est aperçu d'une part que le continent africain semb'ait sérieusement contaminé, d'autre part que l'affection, dans certains cas, avait tendance à progresser dangereusement.

$1^{\circ}$ L'Afrique du Nord n'est pas indemne. On note : 0.79 p. 100 à Alger (25). de I à 15 p. 100 au Maroc, 2,25 p. 100 en Tunisie (13) ef 0,7 p. 100 en Egypte (93).

$2^{\circ}$ En Afrique du Sud, les services sanitaires donnent pour 1956 le chiffre de 2,86 p. 100 . La maladie est pratiquement inexistante au Mozambique ef à Madagascar.

$3^{\circ}$ Pour les autres territoires d'Afrique noire, les écarts dans le degré d'infestation se révèlent sensibles d'un pays à l'autre.

Abyssinie : 80 p. 100. Pour Rocher d'Héricourt. " tous les Abyssins sont affectés du ténia ». Is estiment que la présence du parasite est pour eux un gage de santé.

Kenya : 7,89 p. 100 d'après Mann (58). A Athi-River, d'après Ginsberg (36), 30,6 p. 100 pour le bétail d'origine européenne et 29,4 p. 100 pour le bétail autochtone en 1955 .

Erythrée : 29,9 p. 100 en 1949 (21).

Congo belge (24) :

Ouest et sud, pas de cysticercose.

Elevages indigènes, 64 p. 100 en 1957.

Ruanda : 15 p. 100.

Ituri : jusqu'à 70 p. 100.

Oubangui (87) :

Bangui : 30 à 41 p. 100.

Bouar : 68,6 p. 100 en 1955.

Cameroun (87) : de 15 à 20 p. 100.

Nigéria : environ 10 p. 100 sur le plateau central*.

Niger : 1,5 p. 100 (1957).

Haute-Volta : 6,88 p. 100.

Soudan : 0,78 p. 100.

Guinée : 20 p. 100 (1955).

Guinée portugaise : 7 p. 100 environ (107).

Sierra-Leone : 38 p. 100 (1955).

Sénégal (72-87) : de 0,3 à 2 p. 100.

Mauritanie : 1,2 p. 100.

e) Au Tchad, la répartition de la cysticercose bovine est fonction des régions d'élevage, de l'année et de l'âge des animaux.

$1^{\circ}$ Chez les adultes mâles et femelles stériles de cinq ans et plus, les relevés pratiqués dans les principaux abattoirs de la République donnent les résultats suivants :

a) Abattoir de Fort-Lamy (Ouest : Kanem et Baguirmi). * Communication de $M$. le $D^{r}$ Lee des services vétérinaires de
Nigéria. 
TABLLAU II - CYSTICERCOSE

- Abettorr de Fort-Lamy (Ouest : Kanem et Baguimi)

\begin{tabular}{|c|c|c|c|c|}
\hline & 1954 & 1955 & 1956 & 1957 \\
\cline { 2 - 5 } Animaux abattus & 17.000 & 10.802 & 21.302 & 21.502 \\
Saisies totales * & 23 & 86 & 128 & 99 \\
Pourventages & 0,14 & 0,45 & 0,60 & 0,46 \\
\hline
\end{tabular}

* Ces chiffres ne comprennent pas les langues.

b) Abattoir d'Ati (Centre : Batha).

$\mathrm{De} 1957$ à 1958, 19 animaux atteints sur 208 abattus, soit 9,1 p. 100.

c) Abattoir d'Abéché (Est : Ouaddai).

TABLFAU III - CYOTICERCOSE

Abattoir d'Abéché (Est : Ouaddaï)

\begin{tabular}{|c|c|c|c|c|c|}
\hline & 1952 & 1953 & 1954 & 1955 & $\begin{array}{c}1558 \\
(2 \text { mois })\end{array}$ \\
\cline { 2 - 6 } Animaux tués & 2.224 & 1.555 & 2.198 & 3.063 & 176 \\
Saisies totales & 184 & 71 & 301 & 336 & 25 \\
Fourcentages & 3,2 & 4,5 & 13,2 & 10,9 & 14,2 \\
\hline
\end{tabular}

d) Abattoir de Fort-Archambault (Sud : Moyen-Chari).

Le pourcentage d'infestation oscille autour de 5-12 p. 100 selon les années, les animaux sacri- fiés étant originaires pour la plupart du Batha ou de l'Ouaddaĩ.

$2^{\circ}$ Chez les jeunes, les résultats sont nettement différents tout au moins au Kanem-Baguirmi qui a servi spécialement de champ à nos investigations.

Nous avons eu la possibilité de pouvoir autopsier en quatre ans (1954 à 1957), 912 bouvillons mâles âgés de sept à vingt-quatre mois, en provenance des régions qui nous intéressent (tableau IV).

Le pourcentage moyen chez les jeunes est donc de 15,4 p. 100, chiffre bien supérieur à celui obtenu chez les adultes, même si l'on applique, dans ce cas, un correctif destiné à tenir compte des langues saisies pour cysticercose (qui ne figurent pas dans les statistiques d'abartoir de Fort-Lamy). II n'est pas exagéré de compter pour un adulte ladre, trois à quatre jeunes parasités. Il se révèle assez difficile de donner une explication absolument correcte de cet important écart. Tout au plus peut-on faire remarquer qu'interviennent chez l'adulte divers facteur's tels que le changement de milieu, un mode d'élevage différent, et selon certains auteurs, une résistance acquise de l'individu. 'De' plus, le boeuf de boucherie est tué vers $5-6$ ans, ce qui laisse au jeune un laps de temps suffisant ( 3 à 4 ans) pour se « débarrasser 》 de ses parasites, selon des modalités qui sont loin encore d'être complètement élucidées (dégénérescence puis disparition totale du cysticerque, vu la rareté de la ladre-

TABIFAE IT

Cysticercose chez les jeunes bovins dans l'ouest du Tchad.

\begin{tabular}{|c|c|c|c|c|c|c|c|c|}
\hline & \multicolumn{4}{|c|}{ Kanem } & \multicolumn{4}{|c|}{ Baguirmi } \\
\hline & 1954 & 1955 & 1956 & 1957 & 1954 & 1955 & 1956 & 1957 \\
\hline $\begin{array}{c}\text { Nombre de jeuncs } \\
\text { abattus }\end{array}$ & 117 & 119 & 28 & 186 & 151 & 114 & 100 & 97 \\
\hline $\begin{array}{c}\text { Nombre de jeunes } \\
\text { parasités }\end{array}$ & 26 & 17 & 0 & 24 & 29 & 14 & 11 & 20 \\
\hline Pourcentages & 22,2 & 14,2 & - & 12,9 & 19,2 & 12,2 & 11 & 20,6 \\
\hline Total & \multicolumn{4}{|c|}{$\begin{aligned} \text { Autopsiés } & : 450 \\
\text { Parasités } & : 67 \\
\text { Pourcentage } & : 14,8\end{aligned}$} & \multicolumn{4}{|c|}{$\begin{array}{ll}\text { Autopsiés } & : 462 \\
\text { Parasités } & : 74 \\
\text { Pourcentage } & : 16\end{array}$} \\
\hline
\end{tabular}


rie sèche ?). Comme le faisait déjà observer Peel (73), le taux d'infestation décroit en proportion directe de l'âge de l'animal. Cette constatation faite en Sierra-Leone où les animaux de boucherie sont commercialisés à partir de cinq ans, est valable pour le Tchad et vraisem. blablement pour d'autres pays à élevage de type primitif.

Pour l'Ouaddai et le Batha, nous ne possédons malheureusement pas de chiffres précis. Néanmoins, d'après ce que nous avons pu voir en 1951-52 en Ouaddai sur des animaux destinés à la production de vaccin, le nombre de jeunes de moins de 2 ans touchés par la cysticercose semble très élevé.

\section{$2^{\circ}$ Cysticercus dromedarii.}

Il s'agit pour l'instant d'un parasite essentiellement africain.

En Somalie, on décompte 24 p. 100 de chameaux ef 10 p. 100 de bovins infectés (73): en Erythrée (9-21), 10,55 p. 100 de bêtes à cornes et à Kisimaya (4), 1,5 p. 100.

En Afrique du Sud, il existe de fortes présomptions quant à la présence de Cysticercus dromedarii : Viljoen (114), sans en apporter la preuve formelle, l'aurait recueilli sur un potamochère au Betchuanaland et Martinaglia sur un bceuf à l'abattoir de Johannesburg.

Au Ouaddaī, où nous l'avons découvert pour la première fois en 1951, le cysticerque nous apparaissait comme un parasite des ruminants sauvages ou du chameau, plus rarement du bœuf. Cette façon de penser a été confirmée en 1958 à Abéché où sur 176 bêtes tuées, deux seulement hébergeaient le cestode, ce qui représente 1,I p. 100. Par rapport aux saisies totales, la proportion atteint en gros un animal sur douze.

Nous n'avons jamais eu l'occasion de revoir Cysticercus dromedarii ni au Batha ni dans l'ouest du territoire. Pourtant les hyènes y sont nombreuses ef Taenia hycence abondant à l'autopsie (100 p. 100 au Baguirmi contre 90 p. 100 au Ouaddai).

Le cysticerque n'a pas été décrit jusqu'à présent dans d'autres contrées d'Afrique. II est probable qu'il se manifeste partout où vivent des hyènes en quantité suffisante.

\section{SIEGES DE PREDILECTION}

\section{$1^{\circ}$ Cysticercus bovis.}

On considère classiquement que les muscles et les organes les mieux irrigués constituent les lieux les plus favorables au développement de Cysticercus bovis. On admet que les localisations s'effectuent de préférence dans le cœur. la langue ou les masséters. Or de nombreux auteurs estiment aujourd'hui que ces localisations, tout en demeurant exactes, n'ont pas toute la valeur qu'on a bien voulu leur conférer. Pour certains, la langue et les masséters ne sont pas obligatoirement les premiers touchés (6-25-92). Ailleurs, l'examen du cœur prime tout : de 40 à 80 p. 100 des cas de cysticercose sont mis à jour de cette façon (49-83-102). Actuellement, on préconise de plus en plus l'examen

TABLEAU $V$

Localisations de la cysticercose: jeunes bovins

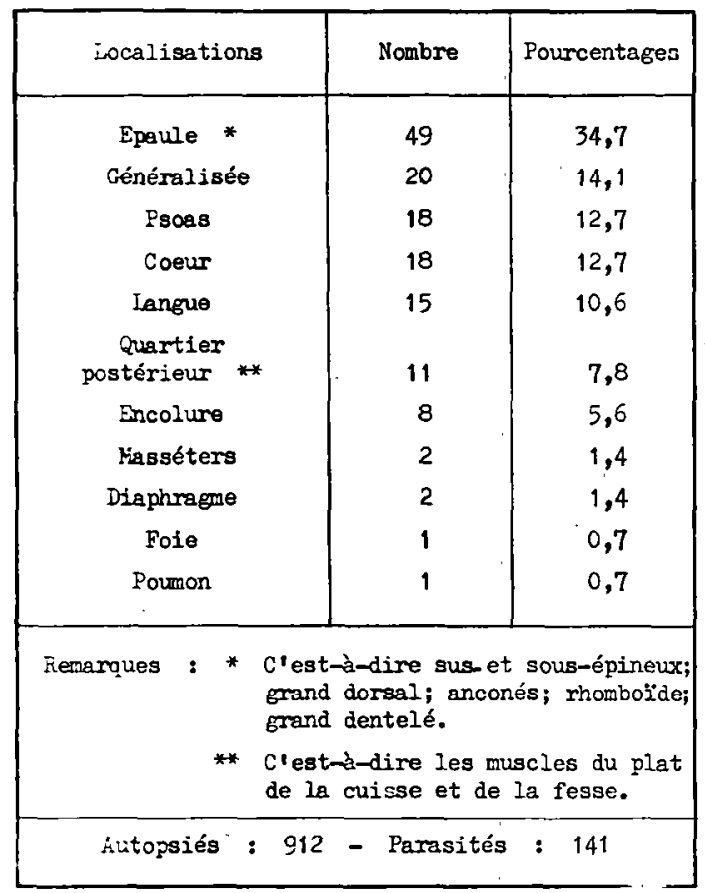

minutieux et systématique des carcasses toutes les fois que la chose est possible (25-38-70-73114). Les résultats semblent bons. Grégoire (38) cite le cas de l'abattoir de Zagreb en Yougoslavie où un examen attentif de 2.342 carcasses a révélé un pourcentage d'animaux infectés de 7.5 p. 100 contre 1 p. 100 auparavant. 
C'est un peu de cette façon que nous avons procédé au Tchad. Les 912 bouvillons étudiés ont été littéralement mis en pièce et sur 14! jeunes reconnus parasités, nous avons relevé les localisations indiquées dans le tableau V.

Pour les adultes, nous n'avons que deux statistiques concernant l'abattoir d'Abéché (tableau VII.

\section{TABLEAU VI}

Abattoir d'Abéché. Localisations de la cysticercose chez les bovins adultes

\begin{tabular}{|c|c|c|c|}
\hline \multirow{2}{*}{ Localisations } & \multicolumn{2}{|c|}{$1954-1955$} & Fév.-mars 1958 \\
\cline { 2 - 4 } & 637 parasités & $\%$ & 23 parasités \\
\hline Langue & 485 & 76,6 & 6 \\
Coeur & 188 & 29,4 & 1 \\
Généralisée & 111 & 17,3 & 6 \\
Foie & 41 & 6,4 & 1 \\
Diaphragne & 33 & 5,1 & 0 \\
Psoas & 21 & 3,2 & 5 \\
Nasséters & 2 & 0,3 & 0 \\
Pounon & 2 & 0,3 & 0 \\
Epaule & & & 12 \\
Quartier & & & 1 \\
postérieur & & & 1 \\
Encolure & & & \\
\hline
\end{tabular}

Dans ces conditions, il est permis de distinguer au Tchad :

a) des localisations essentielles : la langue, l'épaule, le coeur et les psoas.

b) des localisations importantes : membre postérieur et encolure.

c) des localisations secondaires : masséters et diaphragme.

La cysticercose des viscères n'est pas une rareté. Si le poumon ne subit en général qu'une atteinte légère, par contre presque 7 p. 100 des foies, certaines années au Ouaddaĩ, présentent des cysticerques $(1$ p. 100 à peine à Fort-Lamy).

La cysticercose généralisée est assez fréquente : environ 20 p. 100 des adultes à Abéché et 14 p. 100 des jeunes à Fort-Lamy. Ce taux approche 30 p. 100 dans certaines zones du Baguirmi (Massenya - 1957).

A noter également que la partie gauche du corps est plus riche en vésicules que la partie droite, ainsi que le faisait déjà remarquer Reitsma en 1931.

Si l'on compare nos conclusions avec celles qui ont été tirées dans d'autres pays africains. on s'aperçoit que les sièges de prédilection ne sont pas soumis à des variations très sensibles. Au Kenya, Ginsberg (36) donne dans l'ordre d'importance : l'épaule suivie par la langue, le cœur, les masséters, les muscles du membre postérieur, l'oesophage, le diaphragme et les psoas. Par rapport au Tchad, seuls les psoas et les masséters changent de position au classement. Pour Viljoen (114), viennent d'abord les masséters, puis l'épaule, la langue, le cœur, les psoas, les muscles du membre postérieur, le diaphragme. Duvallet (25), tout en se défendan't d'établir une classification précise, n'hésite pas à lever systématiquement les épaules.

La localisation des cysticerques à l'intérieur d'une même carcasse, paraît d'ailleurs assez anarchique. Dans certains cas, il ne se révèlera rien ni sur la langue, ni sur le cœur, les cysticerques n'étant visible qu'au niveau de l'épaule. Ailleurs, il n'y en aura nulle part sauf au niveau de l'articulation du carpe ou au niveau de la langue. Dans d'autres cas, on découvrira un cysticerque sur la langue et par des examens appropriés, plusieurs dans l'épaule ou dans les muscles du plat de la cuisse par exemple. Toutes les combinaisons sont possibles.

Lorsque l'on ne retrouve qu'un seul cysticerque dans une carcasse, il est donc difficile de prétendre qu'il est unique et qu'il n'en existe pas en d'autres points. D'où la nécessité absolue d'examiner très soigneusement les viandes soumises à l'inspection sanitaire.

\section{$2^{\circ}$ Cysticercus dromedarii.}

D'après Pellegrini (72), chez les bovins, les lieux d'élection sont : les ganglions mésentériques, le cerveau, le cour, la langue ef les psoas ; chez le chameau, le foie, le coeur, la langue, le cerveau et les masséters.

Au Ouaddai, on retrouve de préférence Cysficercus dromedarii dans le cœur et les psoas.

La cysticercose généralisée ne semble pas très rare chez les ruminants sauvages : le damalisque 
que nous avons autopsié, en était abondam. ment pourvu (plusieurs centaines).

\section{FREQUENCE DE L'INFESTATION}

Les notions de cysticercose légère ou de cysticercose généralisée n'ont fait l'objet d'aucune codification dans la plupart des législations modernes. Elles sont d'ailleurs très variables d'un pays à l'autre. Au Tchad, la fréquence de l'infestation, paraît être approximativement la suivante :

\section{TADLEAU VII}

Tréquence de la cysticercose au Tchad

\begin{tabular}{|c|r|cc|}
\hline $\begin{array}{c}\text { Nombre de } \\
\text { cysticerques }\end{array}$ & Jeunes & \multicolumn{3}{|c|}{ Adultes } \\
\hline 1 & $20 \%$ & de 10 à $25 \%$ & \\
de 2 à 10 & $50 \%$ & de 50 à $60 \%$ & $\begin{array}{c}\text { suivant } \\
\text { les }\end{array}$ \\
de 10 à 20 & $16 \%$ & de 15 à $20 \%$ & régions \\
Généraisés & $14 \%$ & de 5 à $20 \%$ & \\
\hline
\end{tabular}

Les cas d'infestation très légère (une vésicule) ne dépassent probablement pas 25 p. 100.

\section{INFESTATION PRENATALE}

L'infestation intra-utérine constitue une éventualité qui a été envisagée, il y a longtemps déjà, par de nombreux auteurs (114). Plus récemment, Canham (18) au Natal et Mihajlovic (65); en Yougoslavie, ont signalé la présence de cysticerques sur de très jeunes animaux. Ginsberg (37) en a récolté sur un veau âgé de deux jours : le taux d'infestation, entre le deuxième et le quatorzième jour, se situe, à Athi-River, autour de 4,5 p. 100 . Les dimensions ef l'aspect des vésicules correspondent exactement à ce qui a été décrit par Mc intosh (62).

Au Tchad, nous en avons observé uil cas sur un animal de deux semaines.

L'infestation prénatale impiquue comme conséquence l'examen serré des carcassse de veaux expédiées à l'abattoir, alors que souvent cette inspection est négligée (31-33).

\section{SYMPTOMES}

Les symptômes de la cysticercose bovine sont, jans l'ensemble extrêmement discrets sinon nuls. On a décrit de temps en temps des myocardites caractérisées (94-1/4-116), plus rarement des atteintes cérébrales.

\section{DIAGNOSTIC}

\section{a) Ante Mortem.}

On a tenté de rechercher la cysticercose du vivant de l'animal au moyen de diverses réactions immunologiques (III). Clarenburg (II4), chez le bœuf, utilisant un extrait alcoolique de Taenia saginata a pu obtenir, par la méthode de déviation du complément, certains résultats qui n'ont malheureusement pas été confirmés par tous les auteurs, les réactions étant considérées comme non spécifiques. Par contre la déviation du complément a donné entre les mains d'Ales ot d'Arjona (106) d'assez bons résultats dans le diagnostic de la cysticercose humaine à Cysticercus cellulosae. A partir de l'antigène de Lange modifié, les auteurs parviennent à détecter un grand nombre de cas de cysticercose cérébrale. Ils considèrent qu'une déviation positive consolide singulièrement le diagnostic clinique.

Skvortsov et ses collaborateurs (95) ont mis au point sur le bouf un test cutané. Ils travaillent à partir d'un antigène qui est un extrait de tête d'hydatide en solution salée. Les concilusions ne se sont pas montrées favorables: le taux d'infestation relevé à l'autopsie ne sembie pas coincider avec le pourcentage fourni Dar le test.

Les réactions inmunologiques re deviendront vraiment iritéressantes, en matière de cysticersse bovine, que le jour où elles seront absolums.7t șres et où on connaitra la façon de traiter 'a malaxie : actuellement, un anima'? reconnu de son vivant porteur de parasites, perd de ce fait une grande partie de sa valeur commerciale. Le oropriétaire n'a pas d'autre alternative que de le laisser se stériliser un an ou deux sur un pâturage indemne avec tous les risques que ce genre d'opération comporte c'est-à-dire réinfestation possible, vieillissement ou amaigrissement de la bête, maladies, fraudes, etc. 


\section{b) Post Mortem.}

Les cysticerques sont facilement reconnaissables à l'iniérieur d'une carcasse.

$1^{\circ}$ La diagnose de Cysticercus bovis ne souffre pas de difficulté puisque, seul de tous les cysticerques, it ne possède ni crochets ni rostellum. Pour ce faire, on éclaircit le parasite dûment comprimé, dans de l'acide lactique pendant plusieurj jours. On peut se servir aussi de la méthode de Schmidt-Mulheim qui consiste à placer le cysticerque à examiner à la température de $40^{\circ} \mathrm{C}$ dans 6 à 8 fois son volume d'une solution à I p. 100 d'acide chlorhydrique. Rissling, de son côté, préconise une solution de soude.

Il faudra éviter de confondre les vésicules ladres avec:

a) Les jeures kystes echinococciques : la présence de vésicules proligères renfermant chacune de nombreux scolex lève le doute. De plus, la membrane cuticulaire est blanche, épaisse et stratifiée.

b) Les lésions dues à Sarcocystis hirsuta (Moulé 1887). Ces parasites, de $4 \mathrm{~mm}$ de long sur $3 \mathrm{~mm}$ de large, très tôt calcifiés, se rencontrent dans les psoas et le diaphragme. Au Tchad, la sarcosporidiose est fréquente au Batha 17 p. 100 des bœufs abattus).

c) Les nodules actinomycosiques: l'aspect de la lésion sous le microscope ne trompe pas (grains rayonnés).

d) Les nodules parasitaires répartis çà et là dans la carcasse (foie, épaule, etc.). Provoqués par le passage de divers nématodes, au cours de leurs migrations, ils affectent la forme d'un petit foyer purulent grisâtre entouré d'une coque fibreuse ou d'un gros nodule de $2 \mathrm{~cm}$ environ de couleur verdâtre.

e) Dans la langue, les petits abcès créés par la pénétration d'épines acérées qui peuvent en imposer pour des cysticerques très jeunes.

$2^{\circ}$ Cysticercus dromedarii devra être différencié :

a) D'avec Cysticercus cellulosae dont il est voisin et dont il né diffère, d'après Neveu-
Lemaire, que par le plus petit nombre de crochets (22 à 31) la longueur des plus grands $(160-180 \mu)$ et l'aspect de ces derniers dont le manche est p'us court que la lame.

b) D'avec Cysticercus tenuicollis, dont la loca. lisation est presque uniquement péritonéale, rarement pleurale. Les kystes sont dans l'ensemble beaucoup plus volumineux.

c) D'avec Cysticercus ovis: les crochets sont moins nombreux (24 à 34$)$ et de plus petite dimension (156 à $188 \mu$ pour les grands cruchels et 98 à $128 \mu$ pour les petits).

d) D'avec Cysticercus bovis : l'absence de crochets rend le diagnostic facile. Sur une carcasse, avec un peu d'habitude, on peut se faire une idée du cysticerque en cause, en tenant compte de sa localisation, de la forme de la vésicule et de sa taille, puisque Cysticercus dromedarii atteint assez souvent $19-20 \mathrm{~mm}$.

\section{MODE D'INFESTATION}

\section{1" Cysticercus bovis.}

L'infestation des animaux par Cysticercus bovis dépend de divers facteurs, les uns propres à l'homme, les autres d'ordre physique ou biologique.

\section{a) Les facteurs propres à l'homme.}

Les régions où la cysticercose bovine est la plus répandue sont, en général, celles où les conditions d'hygiène se révèlent médiocres, sinon mauvaises : c'est le fait de beaucoup de pays sous-développés (67-113-117). La contámination des animaux s'effectue directement au moyen de déjections remplies d'cufs ou d'anneaux de Taenia saginata, déjections qui sont rejetées à proximité des zones où vivent les bêtes à cornes : écuries, pourtour des habitations, etc. Dans d'autres cas, l'infestation se produit à partir d'anncaux émis en dehors des défécations normales, puisque l'évacuation du cestode a lieu souvent de cette manière.

Il importe donc que le contact entre le porteur et le bétail soit très étroit : c'est ce qui arrive dans les élevages de type primitif, notamment au Tchad. In brousse, le troupeau, dans de nombreuses régions est rentré le soir dans le but de traire les femelles et d'éviter les vols 
ou l'action des bêtes fauves (lions-hyènes). Ler animaux sont parqués dans des enclos bordés de branches d'épineux ou « zéribas 》 qui correspondent à peu près, du point de vue utilité, aux « kraals » sud-africains ou aux «bomas » du Kenya. Ces «zéribas 》sont construites au voisinage immédiat des habitations. Les éleveurs africains ont l'habitude d'aller défèquer dans ces «zéribas " quand elles sont vides, à l'in. térieur des cases ou même, en dehors des villages, dans les champ de mil et les maigres «bois» qui les ceinturent.

Le bœu ne parait pas naturellement coprophage, mais dans ces pays, comme il manque souvent de protéines et de sels minéroux, il peut le devenir. II ira volontiers lécher, dans les «zéribas», le sable et la terre salée qui supportent les excréments humains. II arrive ainsi à absorber des cufs et des anneaux. Le lendemain matin, le bœuf repart au pâturage, quelquefois fort loin de son lieu de repos.

Les jeunes, de leur côté, sortis des «zéribas» où ils sont soumis aux mêmes conditions que les adultes, s'éloignent assez peu pendant le jour. Au cours de leurs déplacements, ils ont la possibilité de retrouver des déjections humaines émises le matin aux alentours des habitations, dans les champs ou dans la brousse entourant immédiatement le village. Les risques d'infestation sont, dans ces conditions, bien plus grands chez les jeunes que chez les adultes.

Quant aux veaux, ils demeurent attachés près des cases. Dans certaines régions, ils passent même la nuit à l'intérieur de celle-ci, avec leur propriétaire. Cette promiscuité favorise au maximum la cysticercose.

II existe, bien entendu, beaucoup d'autres lieux où bêtes et gens se regroupent. Ce sont les zones de pâture et les puits qui, dans les régions sèches, représentent, durant la journée, un centre d'attraction et d'activité humaine important. Les troupeaux, après $y$ avoir bu, séjournent à proximité, au moment des grosses chaleurs. Les chances d'infestation sont là encore sérieuses.

Dans les villes africaines, on a incriminé les terrains de parcours situées sur le pourtour de celles-ci, terrains généralement transformés en lieu public d'aisance (72).

On a décrit enfin d'autres modes de propagation :
Les voyages : en Amérique, les œufs de Taenia saginata, projetés des toilettes de train, sont susceptibles d'aller infester les animaux qui paissent ie long des voies (5).

Le camping (24) : en Hollande, dans certains endraits, les citadins ont contaminé les pâturage où ils venaient camper.

Le nomadisme : les grands nomades sont capables de disséminer œufs et anneaux sur de très larges surfaces.

\section{b) Les facteurs physiques.}

Leur rôle se situe, paradoxalement, sur deux plans opposés. D'une part, ils agissent en limitant l'infestation, par la destruction des œufs rejetés à l'extérieur; d'autre part, en assurant, dans certaines circonstances, la dispersion de ces mêmes œufs sur une grande échelle.

1" Rôle limitatif.

Des expériences ont été réalisées dans le but de connaitre la résistance des œufs de Taenia saginata. Tous, en effet, ne se transforment pas obligatoirement en cysticerques. Jepsen et Roth (47) ont montré qu'en administrant à des veaux des embryophores au moyen d'une sonde stomacale, il ne restait que 8 à 10 p. 100 de cysticerques avec des doses de 30 à 100 ceufs et 14 p. 100 avec des doses de 500 œufs. Le déchet est donc dès le départ considérable. En tenant compte des facteurs physiques dont nous allons parler, il ne devrait y avoir théoriquement qu'un très petit nombre d'œufs capables de donrer des cysticerques. Malheureusement, le factcur humain (promiscuité, mauvaise hygiène, etc.), joue en modifiant le problème et en laissant aux œufs des chances d'infestation qu'ils ree possédaient pas au départ.

Parmi les facteurs physiques, citons:

a) Le froid. Son action paraît assez faible. En Europe (47), on a pu conserver des embryophores vivants de février à juillet soit pendant près de 23 semaines.

b) La chaleur. Les œufs sont détruits en dix minutes à $59^{\circ} \mathrm{C}$. Ils vivent encore après avoir été chauffés à $45^{\circ} \mathrm{C}$ pendant quatre heures (101).

c) Lo sécheresse semble être le facteur limitatif le plus important. D'après Silvermann (101). les œufs ne survivent pas au-delà de 14 jours, s'il n'y a pas une certaine humidité. 
d) La chaleur et I'humidité. D'après Jepsen (47) au Danemark, des œufs placés dans des touffes d'herbe en juin-juillet, perdent 80 à 90 p. 100 de leur pouvoir infectant au bout de 58 jours. Daubney (22), au Kenya, observe l'infestation d'animaux six mois après avoir fait déposer des anneaux de ténia sur. une petite étendue de pâturage.

e) L'eau. On a eśsayé :

L'eau du robinet $(47)$ à $18^{\circ} \mathrm{C}$. Passé 70 heures, le nombre d'œufs susceptibles de se développer est encore très élevé.

$L^{\prime}$ eau de ruisseau à $18^{\circ} \mathrm{C}$. Les résultats sont à peu près semblables.

L'eau salée (101) à $-4^{\circ} \mathrm{C}$ ef à température ordinaire : les œufs résistent (335 jours dans le premier cas et $60^{\prime}$ jours dans le second).

Les eaux d'égoût à $18^{\circ} \mathrm{C}(47)$ montrent encore un très grand nombre d'œufs viables 19 jours après leur pollution par des anneaux de ténia.

Les purins tuent les cufs dans un délai de 71 jours (47).

\section{$2^{\prime \prime}$ Rôle de dispersion.}

a) La sécheresse. On a remarqué en Africiue du Sud qu'après des périodes de grande sécheresse, on assistait à une recrudescence des cas de cysticercose : l'herbe est, en effet, plus rare, les déjections humaines plus apparentes et les animaux plus affamés.

b) Les eaux.

Les fleuves, les rivières peuvent charrier des œufs de Taenia saginata, parfois fort loin de leur lieu d'origine (113). La chose semble avoir été démontrée pour le Rhin et peut-être pour les régions d'Afrique du Sud voisines de la côte (114).

Les mares, les petits lacs, les eaux stagnantes, souillées par des individus porteurs de parasites, constituent une source do contamination possible pour le bétail qui vient s'y abreuver. Ces collections d'eau, après les pluies, jalonnent les étapes de transhumance et voient passer beaucoup d'animaux, d'où risques d'infestation pour des bêtes indemnes à l'origine.

Les eaux d'égoût, autour des villes, renferment fréquemment des œufs qui ont la possibilité de se maintenir en vie près de trois semaines. Ces eaux alimentent des champs d'épandage em ployés souvent comme pâturages. Les animaux qui y sont placés, sont alors particulièrement exposés à la maladie. Ce mode d'infection a fait l'objet d'études approfondies en Europe et.en Australie ces dernières années (7-47-48-69-9899. 104-1|2-1|4). Jepsen et Roth (48), après avoir installé vingt bêtes sur une pâture de ce type des environs de Copenhague, ont retrouvé à l'abattoir la moitié d'entre elles atteintes de cysticercose.

Les moyens classiques dont on se sert pour purifier les eaux d'égoût (sédimentation, filtre à écoulement lent, digestion anaérabie, boues siccatives), n'empêchent pas le passage des œufs. Par filtration sur sable, il est possible d'en arrêter 50 p. 100 environ (99). La meilleure solution serait, au laboratoire, une sorte de « gaze d'acier. » qui retiendrait 90 p. 100 des œufs.

\section{c) Autres facteurs.}

On s'est aperçu que certains oiseaux tels que mouettes, goëlands, gobe-mouches, qui se posent au voisinage des canaux d'évacuation d'eau d'égoût, pouvaient àvaler des œufs de Taenia saginata, les garder et les expulser au bout d'un certain temps sans quo ceux ci aient perdu la faculté d'évoluer ultérieurement (40-41-99). Ce mode original de dispersion méritait d'être relevé, certains auteurs le considérant comme primordial (en Europe surtout).

\section{$2^{\prime}$ Cysticercus dromedarii.}

Pour que la contamination se produise, il importe qu'un contact étroit soit établi entre le bétail ef les hyènes. Dans lej régions où celles-ci sont nombreuses, cette condition est réalisée autour des villages, autour des puits et sur les lieux de pâture. Les chances d'infestation paraisseirt néanmoins assez faibles.

\section{IMMUNITE}

La question est en pleine évolution et des recherches sont effectuées actuellement dans le but de mettre au point une méthode efficace d'immunisation contre Cysticercus bovis. On sait depuis longtemps déjà (Penfold, 1936 - 1.14) qu'une première infestation détermine dans |'or- 
ganisme de l'animal atteint une libération d'anticorps qui empêchent les œufs de ténia absorbés ultérieurement de se transformer en cysticerques.

On a réussi (17) expérimentalement sur des souris, à provoquer l'immunité contre des cysticerques en migration, à partir de Taenia taeniceformis.

L'immunité croisée (Cysticercus bovis-œufs de Taenia pisiformis) a été mise en évidence récemment (37) et l'on s'oriente aujourd'hui vers l'atténuation des embryophores de Taenia saginata par les rayons $X$.

\section{TRAITEMENT}

On a cherché à traiter la cysticercose bovine au moyen de substances chimiques. Herin et Thienpont (44) injectent à l'animal ladre de l'huile thymolée à $50 \mathrm{p}$. 100 par la voie intra. musculaire. Les résultats se sont révélés décevants : le cysticerque n'est pas tué ; en outre. les délabrements qui font suite à l'injection. apparaissent trop importants pour que la méthode puisse être appliquée sans risque.

Ginsberg (37) de son côté, a observé qu'une solution d'acide oxalique à 0,5 p. 100 était capable in vitro de détruire Cysticercus bovis. L'action de cet acide mériterait d'être précisée in vivo sur des cysticerques adultes et sur des cysticerques en évolution. La question présente un grand intérêt du point de vue économique : le sisal qui pousse en abondance dans certaines région de l'Afrique tropicale, contient, en effet, 0,4 p. 100 d'acide oxalique.

Enfin, d'autres produits qui son't connus comme insecticides et habituellement utilisés comme tels, se montrent très actifs à l'égard de certains parasites gastro-intestinaux. D'après des travaux américains (37), I'action de ces corps pourrait également s'étendre à Cysticercus bovis. Les essais sont en cours.

\section{PROPHYLAXIE}

Nous envisagerons successivement : I. - La destruction des cysticerques.
I1. - L'élimination de Taenia saginata chez l'homme.

III. - Le relâchement de la symbiose hommebétail. La pratique du ranching.

$$
\text { IV. - Le bilan. }
$$

\section{I. - LA DESTRUCTION DES CYSTICERQUES}

Elle sera assurée :

\section{a) Par une inspection sévère des carcasses.}

Le problème de l'inspection des viandes suspectes de ladrerie a déjà fait couler beaucoup d'encre (114). Nous n'y reviendrons pas. Nous nous contenterons de résumer brièvement les principales coupes et incisions préconisées par Vilioen (114), coupes qui semblent les plus appropriées au milieu africain. Ce sont :

Deux longues incisions parallèles dans les masséters de chaque côté de la face.

Deux longues incisions dans les ptérygoidiens.

Quelques incisions dans les múscles de la langue (dans le sens de la longueur).

Palpation manuelle du cœur et incision du ventricule gauche.

Une ou plusieurs incisions dans les muscles de l'encolure.

La levée de l'épaule : celle-ci devrait être systématique dans tous les abattoirs.

L'examen complet des viscères.

Le décollement des psoas avec, au besoin, une incision en longueur dans le muscle.

Une incision dans les muscles adducteurs de ia cuisse, à cinq centimètres de la symphyse pubienne et parallèlement à celle-ci.

L'incision de la bosse et l'inspection de l'œsophage ne semblent pas d'une grande utilité au Tinad.

L'inspection terminée, quelle conduite faut-il adopter ? Là encore les avis divergent. Nous pensons - et bien d'autres avec nous $(36-114)$ que, dans tous les cas, les viandes ladres doivent être retirées de la circulation. quel que soit le degré de l'infestation, faible ou massif. Les moins atteintes seront stérilisées d'une manière ou d'une autre, selon les moyens dont on dispose ; elles seront ensuite remises en vente sous 
le nom de viandes rócupérées ou encore réemployées dans l'industrie de la conserverie. Les plus contaminées seront expédiées à l'équarrissage pour la fabrication de poudres ou d'engrais ou totalement détruites.

Ces mesures draconiennes sont parlaitemenl justifiées : il faut se souvenir que la présence d'un seul cysticerque n'indique pas obligatoirement le degré réel d'infestation, que des cysticerques vivants ef des cysticerques morts peuvent cohabiter et que le rôle d'un abattoir est d'éliminer du circuit commercial les viandes impropres à l'alimentation humaine : les viandes ladres entrent parfaitement dans cette catégorie (64): II serait absurde en effet de vouloir lutter contre la cysticercose en laissant ultérieurement transiter des carcasses qui iront infester d'éventuels consommateurs.

\section{b) Par la multiplication des abattoirs contrôlés.}

C'est une mesure souhaitable, mais lourde d'incidences financières pour des pays sous-développés. L'éleveur, en général, tient à ses bêtes. II lui arrive cependant d'en égorger à l'occasion de certaines cérémonies coutumières, en cas d'accidents, de maladies incurables (peste bovine, péripneumonie, trypanosomiases, etc.) ou en cas de vieillesse, les vieux animaux ne pouvant plus suivre le troupeau.

Cette consommation en dehors de tout contrôle paraît être à l'origine d'un très grand nombre de foyer's de téniasis humain.

\section{c) Par la stérilisation des viandes ladres.}

\section{$1^{\circ}$ Méthodes.}

Elles sont nombreuses. Citons:

a) La chaleur.

C'est le procédé le plus au point. Au Tchad, on le pratique, à l'heure actuelle, dans la plupart des postes de brousse, puisque seuls les abattoirs de Fort-Lamy et de Fort-Archambault sont pourvus de chambre de congélation.

Les travaux d'Allen (2) ont démontré qu'à $54^{\circ} \mathrm{C}$, le scolex du cysticerque s'évagine encore quand il est placé dans une solution de taurocholate de soude et les cellules flamme ont une activité normale. A $56^{\circ} \mathrm{C}$, toute activité cesse et les vésicules sont digérées quand elles passent dans l'intestin de l'homme. Pour plus de sécurité, les services sanitaires américains (89) ont définitivement adopté la température de $60^{\circ} \mathrm{C}$ ou $140^{\circ} \mathrm{F}$ à cœur. D'après Kuchenmeister $(1 / 4)$, uno température extérieure de $77-80^{\circ} \mathrm{C}$ permet, avec d'assez grosses pièces, d'obtenir $63^{\circ} \mathrm{C}$ dans les zones les plus profondes. Pratiquement en tenant compte des aléas d'une telle opération (arrêt ou irrégularité de cuisson), il est conseillé de faire bouillir les viandes pendant une heure au moins à $100^{\circ} \mathrm{C}$.

b) La salaison.

On prépare une saumure à 25 p. 100 dans laquelle les morceaux de viande sont immergés. Pour Clarenburg (114), Cysticercus bovis est tué en cinq jours. Dans la pratique, on attend trois semaines avant d'ôter les viandes de la saumure.

c) Le froid.

Les modalités d'application en sont très diverses et l'on peut presque affirmer qu'à chaque auteur correspond un procédé de réfrigération ou de congélation différent $(51-108-114$; etc.).

Dans les conditions expérimentales, de nombreux essais ont été réalisés à partir de petits morceaux de viande enfermés dans des « freezers » ou des chambres de congélation. Les derniers en date, ceux de Landi et de Monzini (55) sont intéressants. Les auteurs travaillent sur des fragments de 100 à 200 grammes dont l'épaịseur est de 3 à $4 \mathrm{~cm}$. Cysticercus bovis meurt en 9 heures à $-15^{\circ} \mathrm{C}$, en 8 heures à $-20^{\circ} \mathrm{C}$, en 7 heures à $-25^{\circ} \mathrm{C}$ et en 4 heures à $-40^{\circ} \mathrm{C}$. Une chute de $1^{\circ} \mathrm{C}$ retarde le temps de refroidissement de douze minutes environ. Ces expériences confirment/ce que pensait Mönnig : comme le parasite est très bien protégé extérieurement, plus la jongélation est rapide, moins le cysticerque a de chances de résister longtemps.

Dans les conditions commerciales, il n'existe aucune table de correspondance entre la température et le temps nécessaire pour tuer les larves (64). C'est extrêmement regrettable. Aussi les températures envisagées varient-elles considérablement d'un pays à l'autre, sans qu'il soit possible d'en donner une explication satisfaisante : en Allemagne, $-3^{\circ} \mathrm{C}$ d̀ cceur pen- 
dant 24 heures: en France, $-5^{\circ} \mathrm{C}$ pendant 8 jours ou 30 jours à $0^{\circ} \mathrm{C}$; en Italie, $-5^{\circ} \mathrm{C}$ pendant 15 jours: au Tchad, $-12^{\circ} \mathrm{C}$ pendant 5 jours; à Madagascar, $-8^{\circ} \mathrm{C}$ pendant 8 jours (64).

Viljoen (114), à la suite d'expériences probantes, a démontré que, pour une température commerciale de $-10^{\circ} \mathrm{C}$ facilement réalisable, les vésicules de Cysticercus bovis situées en profondeur, sont incapables de survivre plus de sept jours. Les services sanitaires d'Afrique du Sud ont recommandé le passage à la chambre de congélation à $-10^{\circ} \mathrm{C}$ pendant 14 jours. Viljoen estime qu'un délai de 10 jours assure une sécurité totale.

C'est cette méthode qui est aujourd'hui utilisée avec succès en Afrique du Sud, au Kenya (36), au Congo belge (24), en Hollande, au Danemark, en Norvège (64) et en Angleterre (61). Malheureusement, elle présente deux inconvénients : elle est onéreuse, surtout pour des pays africains pauvres; elle nécessite l'immobilisation prolongée des chambres de congélation, ce qui pose des problèmes lorsque la dite chambre est unique et que le nombre de carcasses à congeler est important.

d) Autres mèthodes.

Keller (50) a essayé de traiter les viandes ladres dans une atmosphère de gaz carbonique à $0^{\circ} \mathrm{C} .75 \mathrm{p} .100$ des cysticerques sont détruits au bout de neuf jours et 100 p. 100 le dixième jour. Avec l'oxygène, les résultats paraissent superposables. Plus récemment, Ginsberg (37) a suggéré l'emploi des rayons $X$ et des essais ont lieu actuellement à l'universitó du Michi gan (115). Sous réserve, bien entendu, que les qualités de la viande ne subissent pas de modifications fondamentales, on pourrait envisager l'application rapide de rayons sur des carcasses ladres avant de les remettre dans les chambres de congélation, ce qui réduirait le temps d'exposition à l'intérieur de celles-ci.

\section{$2^{\circ}$ Contrôle de la viabilité des cysticerques.}

Il est indispensable dans un abattoir, et à plus forte raison quand on expérimente une température nouvelle, d'effectuer des tests de via. bilité des cysticerques sur les viandes stérilisées.
Nous les énumérerons brièvement:

a) Modification de l'aspect physique des vésicules.

Le froid ne modifie pas fortement l'aspect du cysticerque. Tout au plus peut-on remarquer, au bout de six semaines de congélation, des changements de couleur, les vésicules les plus superficielles devenant brunes et les plus profondes rosées.

La chaleur a une action plus nette. $A 65^{\circ} \mathrm{C}$, le kyste apparaît flasque, mou, facile à comprimer entre deux lames. L'énucléation est difficile : ie sco'ex, visqueux, se brise facilement.

\section{b) Test à la chaleur.}

Déposé dans de l'eau à $37-40^{\circ} \mathrm{C}$, le scolex s'évagine et s'agite.

\section{c) Coloration.}

Le meilleur produit semble être la solution acide de vert de méthyle. Le scolex des cysticerques morts (chaleur) se colore violemment en vert, tandis que les cysticerques vivants ne prennent pas le colorant.

\section{d) Fluorescence.}

Une fluorescence rouge se manifeste lorsque f'on soumet des cysticerques vivants à l'action des rayons ultra-violets (52). Elle est beaucoup plus faible, sinon nulle avec des cysticerques calcifiés. Ce procédé n'a pas reçu d'applications pratiques.

e) Tests à la bile ef aux sels biliaires.

Pour plus de clarté, nous avons condensé dans un tableau tout ce qui a trait à cette question (tabieau VIII).

Viljoen (114), après avoir rupturé la membrane extérieure du cysticerque, l'immerge dans une solution de taurocholate de soude à 5 p. 100 chauffée à $18^{\circ} \mathrm{C}$. L'évagination du scolex se produit en moins de deux heures. L'auteur fait observer que le procédé est excellent quand on dispose de cysticerques frais; il est déjà moins bon avec des parasites soumis à l'action de la saumure pendant trois semaines ou congelés pendant 24 heures. II est irrégulier dans tous les 
TABIEAU VIII

Viabilité des cysticerques. Epreuves à la bile et aux sels biliaires

\begin{tabular}{|c|c|c|}
\hline Produit utilisé & Température & $\begin{array}{l}\text { Laps de temps nécessaire } \\
\text { à I'évagination du scolex }\end{array}$ \\
\hline $\begin{array}{l}\text { Bile de boeuf pure } \\
\text { (Sachs) }\end{array}$ & $40-41^{\circ} \mathrm{C}$ & 1 à 3 minutes $(*)$ \\
\hline $\begin{array}{l}\text { Bile de boeuf à } 5 \text { p. } 100 \\
\text { (Clarenburg) }\end{array}$ & $40^{\circ} \mathrm{C}$ & de 10 minutes à 8 heures $(* *)$ \\
\hline $\begin{array}{l}\text { Bile diluée } \\
\text { (Malkani) }\end{array}$ & $37^{\circ} \mathrm{C}$ & jusqu'à 20 heures (*) \\
\hline $\begin{array}{l}\text { Solution de taurocholate } \\
\text { de sodium a } 1 \text { p. } 100 \\
\text { (Vialkani) }\end{array}$ & $37^{\circ} \mathrm{C}$ & jusqu'à 18 heures (*) \\
\hline $\begin{array}{l}\text { Solution de taurocholate } \\
\text { de sodium a } 5 \text { p. } 100 \\
\text { (Malkani). }\end{array}$ & $38^{\circ} \mathrm{C}$ & de 29 minutes à 2 heures $(*)$ \\
\hline $\begin{array}{l}\text { Solutions de glycocholate } \\
\text { de sodium à } 1 \text { p. } 100 \text { et } 5 \text { p. } 100 \\
\text { (Halkani) }\end{array}$ & $37^{\circ} \mathrm{C}$ & plus de 20 heures (*) \\
\hline \multicolumn{3}{|c|}{$\begin{array}{l}\text { * = pour des cysticerques frais } \\
\text { des cysticerques passés la la chambre froide }\end{array}$} \\
\hline
\end{tabular}

autres cas (congélation au-delà de 48 heures).

\section{f) Tests d'infection.}

Aussi Vilioen pense-t-il que les tests d'infection selon les méthodes d'Iwanizki ou de Keller, constituent les seuls critères valables. Le principe est le suivant : essayer d'obtenir à partir d'un cysticerque de bœuf l'évagination du scolex dans l'intestin de l'hôte définitif, sans causer de dommage à la santé de celui-ci. Pour ce faire, on place le cysticerque à examiner dans des sacs de soie (Iwanizki) ou des anneaux de celluloïd (Keller). Ceux-ci sont absorbés par un être humain, passent dans l'intestin et sont finalement ramassés pour examens, aussitôt après avoir été rejetés à l'extérieur.

Pratiquement, on se sert d'un tube de celluloïd de $10 \mathrm{~mm}$ de long (diamètre $7 \mathrm{~mm}$, épaisseur des parois $0 \mathrm{~mm}$ 5), ouvert aux deux bouts. Une fine toile de soie est enroulée en une seule épaisseur autour du tube. Les quatre angles de la toile sont alors tordus ensemble, de manière à obtenir deux fils qui seront ćtroitement noućs au corps du cylindre. Les deux morceaux de soie qui couvrent les extrémités du tube vont être tendus comme une peau de tambour.
Le tube ainsi préparé, est avalé par un volontaire. Après élimination, il est ouvert et examiné et sous l'action des sucs digestifs de l'hôte, le scolex s'évagine, s'il est encore vivant.

En définitive, quel test faut-il adopter de préférence ? Il ne faut pas perdre de vue qu'une bonne étude de contrôle doit assurer une sécurité absolue : I'association du test au taurocholate et du test d'infection paraît répondre à cette exigence.

\section{II. - L'ELIMINATION DE TAENIA SAGINATA CHEZ L'HOMME}

\section{ET DE TAENIA HYAENAE CHEZ LA HYENE}

a) L'élimination de Taenia saginata, dans le cas d'un traitement individuel, ne souffre aujourd'hui pas de difficulté. Si les anthelminthiques de type ancien sont de plus en plus délaissés, par contre la pharmacopée moderne offre de nombreuses possibilités : thymol, sels d'étain, dérivés de l'acridine (quinacrine), phosphate de chloroquine, camoquine, diphentane 70, etc.

La prophylaxie de masse est infiniment plus 
délicate à réaliser, surtout dans les pays de transhumance ef les techniques préconisées n'ont pas donné les résultats que l'on escomptait (24||3-1|4).

La première chose à faire est, en collaboration avec le service de santé, d'éduquer le public, en insistant tout particulièrement sur le danger que représente le dépôt des matières fécales à proximité des pâtures des parcs ou des habitations destinées aux animaux. Malgré cela, avec la meilieure volonté du monde, il semble difficile d'empêcher l'émission d'anneaux expulsés en dehors des sel.es normales, anneaux qui iront infester les animaux du voisinage. C'est peut-être le point sur lequel la prophylaxie moderne du téniasis humain risque de buter le plus longtemps (16).

b) La disparition de Taenia hyaenae suppose parallèlement celle des hôtes définitifs : les hyènes. La présence de Cysticercus dromedarii sur le zébu peut fournir un argument supplémentaire à ceux qui sont partisans de leur destruction pour d'autres raisons lattaques et morts d'animaux domestiques). En trois ans (1955-1958). dans les zones montagneuses de l'est tchadien. plus de 3.000 hyènes ont été empoisonnées à ia strychnine et plus de 500 dans le Baguirmi (87).

\section{III. - RELACHEMENT DE LA SYMBIOSE} HOMME-BETAIL - PRATIQUE DU RANCHING

Il est inutile de s'étendre sur les avantages de la pratique du ranching. D'une façon générale, sur un ranch, les animaux sont mieux alimentés, mieux abreuvés, donc en meilleur étał qu'ailleurs. La circulation des êtres humains y est peu intense puisque tout est clôturé. Le personnel de l'établissement lui-même est peu abondant, le rôle des bergers étant limité à certaines opérations courantes /castration, vaccination, déplacements des animaux de parcs à parcs ou vers les points d'eau, etc.).

Toutes ces circonstances, éminemment favorables, laissent supposer qu'en principe le taux de cysticercose devrait étre réduit sur de telles surfaces.

Au Ranch de lOuadi Rimé (Batina), i, a été décidé, pour plus de sûreté, de traiter régulièrement tout le personnel de l'établissement. A la fin du mois, au moment de percevoir les som- mes auxquelles il a droit, chaque employé est tenu d'avaler une dose de quinacrine de 0,80 g. Sur des individus porteurs de Taenia saginata, les essais effectués au laboratoire ont, en effet. montré que le produit se révélait tołalement efficace dans 85 à 90 p. 100 des cas et qu'il était, dans "ensemble, bien supporté par les adultes diète sans purgation).

En opérant de cette façon, l'âge du cestode ne doit pas dépasser 30 jours, s'il y a eu réinfestation dans l'intervalle. Le ténia n'aura environ que 420 à 450 anneaux (86), soit le tiers du chiffre normal. Sa taille ne dépassera pas 2,10 mètres-2,20 mètres (la moitié de la longueur habituelle). Dans ces conditions, les ouffs contenus dans les anneaux, paraissent devoir être peu nombreux, immatures ou incapables de se déveiopper ultérieurement. Les œufs et les anneaux rejetés grâce à l'action mensuelle de la quinacrine. semblent donc se trouver, dès le départ, dans une situation peu adaptée à une évolution ultérieure chez l'hôte intermédiaire.

Les essais commencés, il y a un an, se poursuivent : il faudra attendre, pour tirer des conclusions définitives, que les jeunes nés au ranch soient parvenus au stade de bête de boucherie.

$$
\text { IV. - BILAN }
$$

La lutte contre la cysticercose ne se présente pas comme une opération facile.

Dans les pays riches, notamment les pays européens, il est possible de mettre en œuvre tout à la fois l'inspection rigoureuse des viandes et leur stérilisation, le traitement des individus porteurs - traitement récessaire et accepté et de puissants moyens pour éliminer les sources d'infection (eaux d'épandage). Aussi le taux de cysticercose est-il au demeurant assez peu élevé, avec tendance très nette à la diminution dans certains territoires.

En région sous-développée, le problème est complètement différent. Le milieu humain, la symbiose homme-bétail, jouent un rôle bien plus important qu'ailleurs. Les traitements de masse sont illusoires, vu l'état d'esprit et les habitudes des populations. Actuel'ement, la prophylaxie de la cysticercose bovine est presque uniquement basée sur l'inspection des viandes à l'abattoir. Or le nombre de tueries, danis un territoire 
comme le Tchad, est loin, pour des raisons diverses (faiblesse des abattages, manque de personnel qualifié, coût de l'installation, etc.) de couvrir la totalité du pays. Les moyens de stérilisation restent sommaires et le froid - très onéreux - n'est utilisé que dans les grands centres.

Cette situation peut durer encore longtemps avec tous les ennuis que cet état de choses implique pour un pays producteur ef expéditeur de viandes, indépendamment des conséquences indirectes sur les individus parasités.

Il paraît done souhaitable d'orienter différemment la prophylaxie! Outre les mesures classiques - augmentation du nombre d'abattoirs, généralisation du froid et utilisation moins onéreuse, association des rayons $X-$ il serait intéressant d'employer une méthode capable de déceler le cysticerque du vivant de l'animal. Une bête reconnue atteinte serait alors marquée et traitée, au moyen de produits chimiques. En cas de refus du propriétaire, l'animal serait obligatoirement abattu et stérilisé.

Toutes ces mesures qui demeurent aujourd'hui encore dans le domaine de l'hypothèse, ne pourront être envisagées avec succès que lorsque les méthodes de diagnostic et de traitement seront absolument sûres.

\section{CONCLUSIONS}

a) Il existe au Tchad, sur les bêtes à cornes, deux cysticerques d'aspect à peu près semblable, Cysticercus bovis (Cobbold) et Cysticercus dromedarii (Pellegrini 1945), formes larvaires de Taenia saginata de l'homme (Goeze 1782) et de Taenia Hyaenae (Baer 1924).

b) Cysticercus dromedarii se voit sur les ruminants sauvages (damalisques ef gazelles), les chameaux et les zébus de l'est du pays où le nombre de hyènes est très grand. Nous ne l'avons pas rencontré ailleurs.

Chez le zébu, il faut compter sur un taux d'infestation d'environ 1 p. 100 . Les sièges de prédilection sont le cour et les psoas.

Taenia hyaenae infeste 90 à 100 p. 100 des hyènes abattues. La seule façon efficace de lutter contre ce parasite est d'éliminer le plus possible de porteurs, au moyen d'appâts empoisonnés (strychnine).

\section{c) Cysticercus bovis.}

1" Se rencontre, au Tchad, chez le zébu, chez le chameau (rare), chez le mouton (exceptionnel) et chez les ruminants sauvages qui constituent apparemment une importante source de contamination (gazelles, damalisques, bubales. etc.).

Le taux d'infestation, chez lé zébu adulte, varie considérablement suivant que l'on envisage la zone ouest $(0,40 \mathrm{p} .100)$, le centre $(9,1$ p. 100$)$, l'est (de 4,5 à 14 p. 100) ou le sud du territoire $(5$ à 12 p. 100); chez les jeunes de six mois à deux ans, le pourcentage s'élève à 15,4 p. 100 (de 1954 à 1957). L'écart considérable enregistré entre jeunes et adultes de la même région. semble devoir être mis sur le compte d'une stérilisation progressive - encore mal connue survenant avant que les animaux ne soient vendus pour la boucherie vers l'âge de 5-6 ans.

$2^{\circ}$ Se localise surtout au niveau de la langue, de l'épaule, du cœur et des psoas, moins fréquemment au niveau de l'encolure et du membre postérieur. Les masséters et le diaphragme ne viennent que bien après. Le foie est un organe souvent touché (Ouaddaï). La cysticercose généralisée frappe 14 p. 100 des jeuries (ouest) et environ 20 p. 100 des adultes (est).

$3^{n}$ L'infestation prénalale des veaux est aujourd'hui démontrée.

$4^{\circ}$ Le diagnostic post mortem de la cysticercose ne souffre pas de difficultés particulières. Les réactions immunologiques préconisées ne paraissent pas spécifiques.

$5^{\circ}$ Le mode d'infestation exige un contact étroit entre l'homme, hôte définitif et le zébu, hôte intermédiaire. Cette condition, favorisée par le manque d'hygiène et la méconnaissance du cycle évolutif du parasite, se trouve bien souvent réalisée dans les zones sahéliennes du Tchad à vocation essentiellement paslorale.

Fort heureusement, des facteurs physiques tels que la sécheresse de l'air et les grosses chaleurs, interviennent pour limiter la résistance des œufs, émis par les porteurs humains. Ces mêmes facteurs peuvent jouer en sens inverse, en permettant une dispersion plus ou moins grande des éléments d'infestation (fleuves, mares, eaux d'épandage, etc.). 
$6^{\circ}$ Des essais sont tentés actuellement dans le but de traiter les animaux atteints de cysticercose. L'intérêt du traitement est fonction de la possibilité de mettre en évidence la maladie du vivant de l'animal.

$7^{\circ}$ La prophylaxie nécessite :

a) La destruction des cysticerques.

Par des incisions et des coupes appropriées, il est possible de détecter le maximum de carcasses parasitées. D'une façon générale, toutes les viandes ladres seront retirées de la circulation, définitivement si la cysticercose est massive, provisoirement dans le cas contraire : elles subiront alors une stérilisation par la chaleur, la salaison ou le froid, selon les moyens dont on dispose. Si tous les auteurs sont à peu près d'accord sur la manière d'utiliser la chaleur ou la salaison avec le maximum de sécurité, les avis divergent en ce qui concerne l'emploi du froid. A ce sujet, on ne peut que regretter l'absence d'une table de correspondance entre la température ef le temps nécessaire pour tuer le cysticerque.

b) L'élimination de Taenia saginata.

Individuellement, les ténifuges modernes se montrent efficaces et assez bien tolérés. Par contre, la prophylaxie de masse se heurte à divers obstacles, les uns propres au parasite lui-même (élimination des anneaux en dehors des défécations normales), les autres d'ordre psychologique (interprétation fallacieuse du rôle du cestode) ou matériel (mode d'élevage traditionnel-cherté des anthelminthiques).

c) Le relâchement de la symbiose hommebétail, par la pratique du ranching, forme d'élevage qui est particulièrement recommandée.

d) Malgré toutes ces mesures, la prophylaxie de la cysticercose bovine demeure difficile en milieu sous-développé ef l'on se préoccupe aujourd'hui de renforcer les classiques moyens de lutte par d'autres procédés (diagnostic du vivant de l'animal - traitement) qui sont d'ailieurs loin d'être au point).

Section d'he/minthologie Laboratoire de recherches vétérinaires de Farcha, Fort-Lamy - Tchad.
BIBLIOGRAPHIE

I. ALADEL (1943). - Thèse vétérinaire. Toulouse.

2. ALLEN, R.W. (1947). - J. Parasit. 33 (4), $331-8$.

3. ALVES DA CRUZ, A. (1955). - Bull. Off. intern. Epiz., 43 (1-2), 217.

4. ANGELOTTI, S. (1947). - Boll. Soc. ital. Med. Ig. trop., 7 (5-6), 544-9.

5. Anonyme (1954). - J. amer. Med. Assoc., I54 (1). 102.

6. Anonyme (1957). — Vet. Rec., 69 (4), 66.

7. Anonyme (1957). - O.M.S. Sér. Monog., 33, 484.502.

7'. BAER, J.G. (1924). - Ann. Parasit. hum. COMP., 2, 247.

8. BAER, J.G. (1926). - 11th and 12th Rep. Dir. Vet. Ed. Res., (1), 63-136.

9. BATELLI, C. (1949). - Boll. Soc. ital. Med. Ig. trop., 9 (3), 289-94.

10. BIRKET,, J.D. (1953). - Vet. Rec.; 65 (24), $391-2$.

II. BLACK, D.A.K. (1955). - Lancet, 2 (6883), 253.

12. BRIZARD, H. (1953). - Rev. Elev. Med. vét. Pays trop., 6 (1), 9.

13. BRUMPT, E. (1949). - Précis de parasitologie. Paris.

14. CADEAC, C. (1914). - Encycl. périod. Pathologie interne, 92.

15. CALL, C. (1949). - Boll. Soc. ital. Med. Ig. trop., 9 (3), 300-2.

16. CAMERON, T.W.M. (1953). - Proceed. $X V$ th. intern. vet. Congr. Stockholm, 261.

17. CAMPBELL, D.H. (1938). - J. Immunol., 35, 465.

18. CANHAM, A. (1946). - J.S. Afr. vet. Med. Assoc., 17 (3), 169-71.

19. CASAROSA, L. (1950). - Clin. vet. Milan. 73 (2), 33-8.

20. CHENGE, P. (1958). - Bull. Off. intern. Epiz., 49 (bis), 466-7. 
21. COCEANI, C. (1949). - Boll. Soc. ital. Med. Ig. trop., 9 (3), 295-9.

22. DAUBNEY, R. (1944). - Rep. vet. Dept. Kenya, 15-6.

23. DESCHIENS, R. ef RENAUDET, R. (1911). - Bull. Soc. Path. exot., 34 (1-3), 17-25.

24. Discussion : Rapport Merle (1958). -- Bull. Off. infern. Epiz., 50, 682-90.

25. DUVALLET (1956). - Rev. Méd. vét., 19 (2), $1 \mid 6-21$.

26. ENDRIJAT, E. (1938). - Deutsch. tierärztl. Wochens., 46 (30), 472.

27. EUZEBY, J. (1957). - Rev. Elev. Méd. vét. Pays trop., $10(1), 19$.

28. FAUST, E.C. (1955). - Animal agents and vectors of human disease. Philadelphia 327 32.

29. FONTENEAU, M. (1950). - Rec. Méd. vét., I 26 (6), 35I-5.

30. FOURNIER, P.N. (|861). - Thèse médicale. Paris.

31. GAEDTKE (1955). - Monatsheft. für Veterinärmed., 12, 277-8.

32. GAILIUNAS, P. (1957). - Vet. Med., 58 (8), 379-81.

33. GEBAUER, O. (1951). - Wien. tierärztl. Monats. 38, 578-80.

34. GINSBERG, A. (1954). - E. Afr. Med. J., 31 (3), 8I-8.

35. GINSBERG, A. (1955). - E. Afr. Agric. J., 20 (4), 216-9.

36. GINSBERG, A., CAMERON, A., GODDARD, W.B. ef GRIEVE, J.M. (1956). Bull. Epiz. Afr. (I.B.E.D.), 4 (I-2), 27-39.

37. GINSBERG, A. (1958). - Bull. Epiz. Afr. (I.B.E.D.), 6 (2), 190.

38. GREGOIRE, C., GRANVILLE, A., POUPLARD, L., DEBERDT, A., SPRENGERS, R. ef VIlLLANYl, J. (1956). - Ann. Méd. vét., 100 (I), 24-36.

39. GRIFFITHS, R.B. (1950). - Ann. trop. Med. Parasit., 44 (4), 357-60

40. GUILDAL, J.A. (1956). - Nord. Veferinaer.,
8, 727-33.

41. GOTSCHE, N.O. (1951). - Nord. vet. Med. 3, 957.

42. HAGEN, K. (1955). - Berl. Tierärztl. Wochens., 68 (2), 27.

43. HARDWICK, E.F. (1950). - Vet. Rec., 62 (45), 633.

44. HERIN, V. et THIENPONT, D. (1957): Ann. Méd. vét., IOI (2), 141-6.

45. HIGGINS, R.H.C. (1940): - E. Afr. Med. $J ., 16(12), 480-1$.

46. HOUDEMER, E.F. (1938). - Recherches de parasitologie comparée indochinoise Paris.

4.7. JEPSEN, A. and ROTH, H. (1952). Proceed, 14th. intern. vet. Cong. London, 43-50.

48. JEPSEN, A. and ROTH, H. (1950). - Nord. Veterinaer., 2 (11). 967-91.

49. JUMP, A. (1949-55). - Ann. Rep. Med. Off. Hlth. Letchworth.

50. KELLER (1938). - Zeitschr. für Fleisch und Milchiyg., 49 (3), $43-5$.

51. KELLER, H. (1938). - Zeitschr. für Fleisch und Milchhyg., 48 (17), 322-5.

52. KOLLER, R. (1943). - Zeitschr. für Fleisch und Milchingg., 53 (19), 185-6.

53. KUPEY, P. (1954). - Wissensch. Zeitschr. Berlin, I, 89-110.

54. KURTPINAR, H. (1955). - Bull. Off. Intern. Epiz. 43 (1-2), 287.

55. LANDI, A. et MONZINI, A. (1954). - Clin. vet. Milan, 77 (9), 264-8.

56. LEPES, T. (1954). - Hiijiena Belgrade, 6 (2). 178-85.

57. LEUCKART, R. (|886). - The parasites of man. Edinburgh.

58. MANN, I. and MANN, E. (1947). - Brit. vet. J., 103 (7), 239-5!.

59. MAROTEL, G. (1949). - Parasitologie vétérinaire. Paris, 116-7.

60. MARQUARD, W. (1938). - Zeitschr. für Fleish und Milchhyg., 48 (23), 44.4. 
61. Mc CLEERY, E.F. et BLAMIRE, R.V. (1950). - Vet. Rec., 62 (33), 477-9.

62. MC INTOSH, A. (1956). - J. Parasit., 42 (4), sect. 2, 41.

63. MENDOZA, M.A. (1940). - Rev. San. Assis. soc. Caracas, 5 (3), 47)-80.

64. MERLE, A. (1958). - Bull. Off. intern. Epiz., 49 (7-8), 483-500.

65. MIHAJLOVIC, S. (1952). - Vet. Glas. Belgrade, 6 (9-10), 574-48.

66. MITROFANOV, V.M. (1957). - Veterinoryia, Moscou, 34 (5), 72-4.

67. MONNIG, H.O. (1941). - J.S. Afr. Vet. Med. Assoc., 12 (2), 59-6).

68. NEVEU-LEMAIRE, M. (1936). - Traité d'helminthologie médicale et vétérinaire. Paris.

69. NEWTON, W.L., BENNETT, J. ef PHILIPS, M. (1949). - Amer. J. Hyg., 49, 166.

70. OLAF NIELSEN (1946). - Medlemsb/. Danske Dyrlaegeforen., 28, 152.

7I. PARDI, M.C., DUARTE, G.G. et ROCHA, U.F. (1952). - Riv. Fac. Med. Vet. SâoPoulo, 4 (4). 613-28.

72. PARLIER (1938). - Rev. Méd. vét., 90, 504-15.

73. PEEL, E. (1953). - Vet. Rec., 16, 244.

74. PELLEGRINI, D. (1942-5). - Rac. Stud. Ric. Patol. Vet. Somaliland, I, 42-8.

75. PELLEGRINI. D. (1942-5). - Rac. Stud. Ric. Potol. Vet. Somaliland, I, 49-52.

76. PELLEGRINI, D. (1947). - Boll. Soc. ital. Med. Ig. trop., 7 (5-6), 544-9.

77. PELLEGRINI, D. (1947). - Boll. Soc. ital. Med. Ig. trop., 7 (5-6), 564-5.

78. PELLEGRINI, D. (1947). - Boll. Soc. ital. Med. Ig. trop., 7 (5-6), 566-72.

79. PELLEGRINI, D. (1948). - Boll. Soc. itol. Med. Ig. trop., 8 (3-4), 172-5.

80. PELLEGRINI, D. (1949). - Boll. Soc. ital. Med. Ig. trop., 9 (3), 284-8.

81. PELLEGRINI, D. (1950). - Bull. Off. intern. Epiz., 33 (1-2), 21-7.

82. PELLEGRINO, A. (1954). - Progress. Vet. Torino, 9 (5). $174-6$ et $178-80$.
83. PENFOLD, W.J., PENFOLD, H.B., ef PHILIPS, M. (1938). - Med. J. Aust., I (3). $107-13$.

84. POPOVIC, M. et VUKONIC, V. (1954). Vet. Sarajevo, 3 (1). 143-6.

85. PRIESTLEY, H. (1950). - Vet. Rec., 62 (38), 569-70.

86. RAILLIET, A. (1895). - Traité de zoologie médicale ef agricole. Paris.

87. Rapp. ann, divers. (Tchad, Cameroun, Oubangui, Nigeria, A.O.F., etc.).

88. RAUST, R. (1952). - Bull. Acad. vèt. France, 25 (3), 113.5.

89. Rep. Bur. anim. Ind., U.S. Dpt Agric., 1946, 47, 113-23.

90. REUTER, F. (1944). - Zeitschr. für Fleisch und Milchhyg. 54 (16), 158-9.

91. RIBEIRO P. de ASSIS. (1949). - Rev. Fac. Med. vet. Saô-Paulo, 4 (I), 167-83.

91'. ROGOSIC, D. (1955). - Vet. Glas. Be/grade, 9 (II), 807-9.

92. SEILER, H.E. et NORVAL, J. (1950). Hith. Bull. Dpt Hlth. Scotland, 8 (3), 46-7.

93. SHERAF EDDIN (1955). - Bull. Off. intern. Epiz., 43 (1-2), 207.

94. SENEVIRATNE, R.D. ef KULASIRI. C. (1950). - Brit. vet. J., 3 (9), 387-90.

95. SKVORTSOV, A.A., SOKOLOVA, L.N. ef TALIZIN, F.F. (1941). - C.R. Acod. Sci. U.R.S.S. 32 (7). 523-5.

96. SILVERMANN, P.H. (1955). - Ann. trop. Med. Parasit., 49 (4), 429-35.

97. SILVERMANN, P.H. ef GRIFFITHS, R.B. (1955). - Trans. roy. Soc. trop. Med. Hyg.. 49 (1). 8.

98. SILVERMANN, P.H. (1955). - Adv. Sci. London, 12 (45), 108-11.

99. SILVERMANN, P.H. ef GRIFFITHS, R.B. (1955). - Ann. trop. Med. Parasit., 49 (4). 436-50.

100. SILVERMANN, P.H. (1956). - Trans. roy. Soc. trop. Med. Hyg., 50 (1), 8.

101. SILVERMANN, P.H. (1956). - Trans. roy. Soc. trop. Med. Hyg., 50 (I), 8. 
102. SILVERMANN, P.H. (1956). - Trans. roy. Soc. trop. Med. Hyg., 50 (1), 7.

103. SUMMA, H. et STEPPE, W. (1956). Tierörztl. Umsch., II (9), 31I-9.

104. SWIERSTRA, D. (1955). - - Tïdschr. V. Diergeneesk., 80 (14), 647-55.

105. TALAVERA, J. (1955). - Bull. Off. intern. Epiz., $43(1-2), 217$.

106. TALAVERA, J. (1957). - Bull. Off. intern. Epiz.; 584-604.

107. TENDEIRO, J. (1947). - Riv. Med. vet. Lisboa, 42 1(321), 1.28-86.

108. TERHORST, H. (1938). - Dissertation. Giessen, pp. 32.

109. THORNTON, H. (1952). - Textbook of meat. inspection. $2^{e}$ édit. London.

1 10. THORNTON, H. (1957). - Vet. Rec., 69 (5), 102.
III. TRAWINSKI, A. (1957). - Bull. Off. intern. Epiz., 191-7.

112. VASIKOVA, Z.G. (1944). - Medi Parasit. Moscou, 13, II.

113. VERSYCK, $M$. et JACOB, H. (1958). Bull. agric. Congo belge, 49 (1), 155-63.

114. VILJOEN, N.F. (1937). - Onderstepoort J. Vet. Res., 9 (2), $337-570$ (importante bibliographie).

I15. VILLELA, S.E, et' coll. (1957). - Amer. J. Path., 3, 619:

116. WADDINGTON, F.G. (1945): - Vet. Rec., $57(31), 366$.

117. WINTHERHALTER, M. et STUPARIC, D. (1957). - Vet. Glas. Belgrade, 2 (4), 458. 65.

118. X... (1958). Presse méd., 66, 596.

\section{SUMMARY}

\section{Bovine cysticercosis}

\section{Its importance in the Saharian production areas of the Tchad Republic}

The author records the presence in cattle of that area of Tchad Republic within - the Sahara of iwo related species of Cysticercus, the descriptions of which he repeats viz. $C$. bovis (Cobbold) and C. dromedarii (Pellegrini 1945), the larval forms of Taenia saginata (Goeze 1782) of man and $T$. hyaenae (Baer 1924) of the hyena.

The incidence of $\mathrm{C}$. dromedarii is about $1.1 \%$ in the zebu of the eastern part of the territory with predilection sites in the heart and psoas muscles.

The incidence of $C$. bovis is adult cattle varies considerably between regions $(0.4 \%$ to $15 \%)$. In young cattle in the West of the territory the incidence is $15 \%$.

The predilection sites of $\mathrm{C}$. bovis are in order of importance tongue, shoulder, heart, psoas, neck, hind leg, masseter muscles and diaphragm.

Further studies are being undertaken on questions of prenatal infection, diagnosis, treatment, immunity and prophylaxis of bovine measles. The heavy annual economic loss from this infestation in under-developed countries necessitates the improvement of the classical measures employed for the reduction of this zoonosis.

\section{RESUMEN}

\section{La cisticercosis bovina}

\section{Su importancia en las zonas de cria de la República del Tchad}

El autor señala la existencia en el ganado de las zonas de cria de la República del Tchad de dos cisticercos parecidos; de los cuales recuerda la descripción, Cysticercus bovis (Cobbold) y 
Cysticercus dromedarii (Pellegrini 1945) formas larvarias de Taenia saginata (Goeze 1782) del hom: bre y de Taenia hyoenae (Baer 1924) de la hiena.

Cysticercus dromedarii afecta alrededor de 1,1 p. 100 de los zébus de la parte oriental del territorio, teniendo como localización esencial é corazón y los psoas.

El porcentaje de infestación por Cysticercus bovis en los adultos sufre variaciones sensibles según las regiones (de 0,4 a 15 p. 100). En los jóvenes, alcanza 15 p. 100 en el oeste del territorio.

Las localizaciones de predilección son, en orden de importancia : la lengua, la espalda, el corazón, los psoas, el cuello, los miembros posterioses, los maseteros y el diafragma.

El autor, sirvíendose de los más recientes trabajos estudia, además, un cierto número de cuestiones tales como la infestación prenatal, el diagnóstico, el tratamiento, la inmunidad y la profilaxis de la cisticercosis bovina. Señala especialmente la necesidad, en pais subdesarrollado, de completar los medidas clásicas de lucha contra esta zoonosis cuyas incidencias económicas son cada año muy importantes. 